\title{
An Algorithm for Adverbial Aspect Shift
}

\author{
Sabine Gründer \\ Department of Computer Science, University of Leipzig \\ Johannisgasse 26, 04103 Leipzig, Germany \\ gruender@uni-leipzig.de
}

\begin{abstract}
The paper offers a new type of approach to the semantic phenomenon of adverbial aspect shift within the framework of finitestate temporal semantics. The heart of the proposal is a supervaluational concept of underspecification, and the idea of treating the meanings of temporal prepositions as dynamic presuppositions. The simple shifting algorithm used in the present approach derives the correct set of possible readings on the basis of lexical semantic input only, and, furthermore, may claim cognitive plausibility.
\end{abstract}

\section{Introduction}

The referents of temporally determined sentences are usually talked of as situations. The traditional ontological classification schema (Vendler, 1957) divides up the respective situational types into at least four abstract subclasses: states, activities, accomplishments and achievements. This aspectual taxonomy takes into account the internal temporal structure of the situations, and is based on a small number of features, such as: telic, dynamic, durative.

Situations are referred to by verbal phrases. Differences in truth conditions, presuppositions and entailments of the respective sentences, as well as effects on temporal sequencing in discourse are plausibly built on the aforementioned structural differences. That the assumed ontological aspectual classification is reflected on linguistic level is (at least partly) borne out by a number of language

(c) 2008. Licensed under the Creative Commons Attribution-Noncommercial-Share Alike 3.0 Unported license (http://creativecommons.org/licenses/by-nc-sa/3.0/). Some rights reserved. internal testing procedures, such as grammaticality effects in connection with imperative, progressive, pseudo cleft and adverbial constructions.

The aspectual character of a verbal phrase is not solely fixed on the basis of verbal lexical information but can change during the process of semantic composition under the influence of nominal arguments and prepositional phrases, tense and aspectual markers, aspectual auxiliaries, or temporal adverbials. Accordingly, the aim of a theory of aspect is to determine the aspectual characteristics of complex verbal phrases on the basis of semantic information associated with certain of its parts.

In the last thirty years, considerable progress has been made in formally describing the aspectual contribution of nominal and prepositional arguments. However as far as the aspectual behavior of temporal adverbials is concerned, some puzzling sets of data still await proper explanation and appropriate formalization. The observation that problems arise right at that point gets particularly worrying given that the very same types of examples are standardly used as linguistic test cases to differentiate telic from atelic verbal phrases. Therefore, if we could find significant problems with the theory just here, the whole idea of a language internal criterion for the division of verbal expressions according to the aspectual situational schema would suddenly become vague and ungrounded.

The troublesome examples under discussion test for compatibility of a verbal phrase with "for" and "in"-adverbials. According to observational facts - so the traditional story goes - atelic states and activities admit modification by a "for"-adverbial, but give rise to ungrammaticality when combined with an "in"-adverbial. For telic achievements and accomplishments things are precisely the other 
way around. The data under (1) and (2) shortly repeat this well-known pattern.

\section{(1) be quiet for an hour /*in an hour swim for an hour / *in an hour}

\section{(2) arrive *for an hour / in an hour} build a tower *for an hour / in an hour

However, after closer inspection, these claimed test cases do not seem to be as simple and clear cut as one would like, given their fundamental theoretical status. The marked phrases, far from being ungrammatical, quite naturally display several kinds of meanings. The focus of the "in"-temporal adverbial under (1) can be read as being shifted from the situation itself towards its pre-phase, which is to lead up to the swimming process or to the state of being quiet only an hour later than the present. In cases where an implicit boundary for the activity is given by context (e.g., a triathlon competition) the process itself can be also modified by the adverbial. For the telic examples in (2), an iterative interpretation appears to be available, with the "for"-adverbial giving the duration of the whole complex situation rather than just of one of the included basic events. Another interpretational possibility here is to let the adverbial fix the duration of the preparatory phase, i.e., when the change is under development, which precedes the culmination of the situation. Finally, the "for"-adverbial is also able to modify the result state following the culmination of the event.

These phenomena in connection with the application of temporal adverbials to situations that are - under the perspective of traditional aspectual theory - not suitable for them have proven to be notoriously difficult to treat in every kind of available framework. At the same time, the observed cases do not have the status of real exceptions, but appear cross-linguistical and spread over the whole verbal lexicon. Therefore, no theory of aspect can be seen as fully adequate as long as it is not able to account for this flexibility in adverbial semantics. The aim of this paper is to work out a semantic analysis of the cases in question which allows to derive the correct set of possible readings in a simple and fully compositional manner. Additionally, the proposal will aim at explaining the underlying semantic mechanism in terms of a more general cognitive principle.

\section{The State of the Art}

Before turning to my own proposal for an analysis of aspectual meaning shifts in connection with the interpretation of temporal adverbial modifiers, let me briefly discuss the idea figuring prominently in virtually all recent approaches, e.g., (Pulman, 1997), (deSwart, 1998). I am going to argue that by following this general line of thought too strictly, linguistic research so far failed to answer the question about the source of the semantic flexibility of temporal adverbials (compare (Gruender, 2008)). The claim to be made in this section is that the problems one can point out come about inevitably and for principle reasons. And that they should be seen as the result of an attempt to take a good metaphor too literally.

Moens and Steedman (1988) conceived temporal adverbials as "functions which 'coerce' their inputs to the appropriate type, by a loose [sic!] analogy with type-coercion in programming languages". Under this perspective, aspectual shift is triggered by a conflict between the aspectual type of the situation to be modified and the aspectual constraint set by the temporal preposition heading the modifier. ${ }^{1}$ Coercion operators, then, are thought to adapt the verbal input on the level of model-theoretical interpretation by mapping one sort of situation onto another. The underlying model is commonly supposed to include situations as first-order objects of the four basic types previously mentioned, i. e., states, activities, accomplishments and achievements.

A first problem is immediately evident with this approach. Since the temporal prepositions constrain the aspectual type of the situation only as far as telicity is concerned, the relation established by coercion cannot be functional. With there being no types telic and atelic but just two more specified primitive types for each case, which are fulfilling the respective aspectual requirement equally well, application of coercion inevitably leaves one with several possible outputs. Since, on the other hand, in examples of real type-coercion, as applied in programming languages, the introduced relation is always functional, a first clear, formal difference

\footnotetext{
${ }^{1}$ Accordingly, the approach is not going to treat examples (i. e. "hiccup all day") where aspectual shifts appear to follow solely from conceptual background knowledge concerning default durations of certain sorts of events, rather than being triggered by a formal semantic conflict in aspectual type requirements. Likewise, I will disregard the possibility of generic interpretations.
} 
between the operations in the two domains has to be noted. A simple mapping between two primitive situations cannot be what is formally going on in cases of meaning shifts triggered by temporal adverbials, as the relevant formal criterion is too general as to effectively constrain the output type.

This problem becomes even more serious when we take into account the variety of aspectual shifts that actually occur according to the previously given examples. There it becomes clear that even if confined to one of the assumed primitive ontological sorts, the output value of the operation would still remain underspecified. So, for instance, after applying a "for"-adverbial to an accomplishment, the iterative and the result state interpretation count as stative alike. According to the type-coercion paradigm, however, in order to remedy ungrammaticality just one such type-adapted output value would have been sufficient. The fact of there being this exact number and kinds of readings therefore becomes a miracle, which the theory is unable to explain and formally account for.

On the one hand, when the telicity/atelicity constraint is associated with the temporal adverbial in the known way, it generally seems to be too loose with respect to the observed transitions, at the same time it appears to be too restrictive. In the boundary reading, the adverbial is successfully applied to a situational concept carrying the wrong telicity feature without any visible aspectual shifts occuring. At this point the type-coercion paradigm obviously is as close as it could be to being led into contradiction.

How do these very general problems present themselves within the known theoretical approaches? In the course of semantic composition coercion operations get introduced as some kind of metavariables. These variables are instantiated by a variety of concrete operations which relate the input situation to an output situation in all the ways possible. The answer to the decisive question of which functions actually are the ones possible here, however, is just read off the data. From theory alone nothing beyond the telicity/atelicity requirement for the output value would follow, and even this prediction is not strictly borne out, as was argued in the previous paragraph. Therefore, the solution has just been given by empirical observation rather than derived through any theoretical systematization. Here, semantic formalization has become a mere record of the data without further explanatory power.

Furthermore, by having set theoretical focus on the ontological level when implementing aspectual shifts, linguistic research has taken up a perspective entirely extrinsic to language and the adaptive powers of meanings themselves. In result of following the type-coercion metaphor, language comprehension is seen as proceeding via an implicit series of program clashes, only to be repaired by simply not obeying to compositional rules for combining lexical semantic information any longer. However, according to a linguistically inspired, representational view, the manipulations at work in case of aspectual shifting would take place within the realm of Fregean sense, rather than that of reference. The denotation of a situational expression so would constantly get re-computed, as semantic composition proceeds, via the systematic modification of its meaning. By not only involving ontological type information but also semantic information proper, such transition process should effectively determine the denotation of the respective output situational concept in the end, and therefore deliver the theoretical derivation of the aspectual shifting operations that we are after.

Summarizing the result of this section, I claim that the analogy between aspect shift as triggered by temporal adverbials and type-coercion as known in the programming language domain is not as close as generally assumed, and surely does not recommend itself for direct implementation. Applying the formal mechanism used in the latter case too strictly to the former, inevitably leads to the interrelated problems mentioned, and seems by now to obstruct a deeper understanding of what is actually going on when senses change and adapt of their own accord.

\section{The Framework}

In this section, I will shortly introduce a decompositional approach to event semantics within the framework of finite-state temporal semantics developed by Fernando (2003), (2004). The decision to apply this kind of formalism in the present context was motivated by the intuition that what was needed to appropriately handle aspectual semantic phenomena was the concept of a situation as a complex but logically coherent, dynamic unit. That means, if aspectual features are defined with respect to some internal, temporal structure of a situation, and if aspectual transitions proceed via 
shifting focus of an adverbial along the time line from one part of that structure to another, this very structure must be represented by the formalism, and has to be made easily accessible by the formal operations. Obviously, the traditional Davidsonean event semantics, where events are seen as indivisible atoms, and modifiers as just indifferently predicating over such atoms, is not a convenient formal tool in this respect.

In Fernando's approach a situational concept, is characterized as a regular language, accepted by a finite-state automata, that may amount to a finite Kripke model with partial valuations. Each string of such language is viewed as a temporal sequence of observations or a comic stripe. The symbols in the alphabet of the language are the still-pictures or snapshots to be strung together in chronological order. Given a finite set $\Phi$ of formulas, or fluents, a symbol consists of a consistent subset of $\Phi$, which non-exhaustively enumerates what holds true at some single point in time. Therefore, a situation is represented not just as an atom, but all its internal states are taken into account as well.

A suitable compositional device for stepwise construction of complex situational concepts is available in form of a regular operation superposition (\&) over two languages ( $\left(\mathrm{L}\right.$ and $\left.\mathrm{L}^{\prime}\right)$. Superposition performs componentwise alignment of strings of equal length and is realized model-theoretically as conjunction.

$$
\begin{aligned}
\mathrm{L} \& \mathrm{~L}^{\prime}=\bigcup_{k \geq 1}\left\{\left(\sigma_{1} \cup \sigma_{1}^{\prime}\right) \ldots\left(\sigma_{k} \cup \sigma_{k}^{\prime}\right)\right. \\
\left.\mid \sigma_{1} \ldots \sigma_{k} \in L, \sigma_{1}^{\prime} \ldots \sigma_{k}^{\prime} \in L^{\prime}\right\}
\end{aligned}
$$

A simple example (by Fernando), deriving the representation for the phrase "rain from dawn to dusk", should make clear the general idea ( stands for $\emptyset$-as-a-symbol, boxes replacing braces).

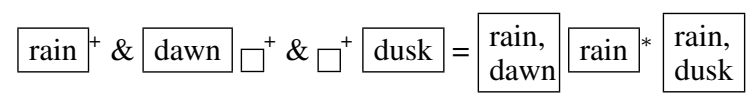

Negation of symbols is defined in De Morgan style by:

$$
\neg \square=\Phi ; \neg \phi_{1}, \ldots, \phi_{n}=\neg \phi_{1}+\ldots+\neg \phi_{n}
$$

(writing + for non-deterministic choice).

Fernando's central idea for a definition of aspectual features is to formally base it on the symbols $\alpha$ and $\omega$ that start and finish a given language, respectively. This serves to enable a nice formal encoding of the idea of a situational type being initially or finally bounded or unbounded. If the condition $\alpha$ is immediately switched after the first stage an initial boundary is marked; if $\alpha$ is preserved the concept is initially unbounded. In the same way $\omega$ can be used to mark a final boundary. Aspectual features then just enumerate all the possibilities for a corresponding concept to be bounded or unbounded in that sense.

$$
\begin{array}{lll}
\text { telic }(\mathrm{L})= & \neg \omega(\mathrm{L})^{+} \\
\text {iter }(\mathrm{L})= & \quad \omega \omega(\mathrm{L})^{+} \\
\operatorname{prog}(\mathrm{L})= & \square \neg \alpha(\mathrm{L})^{+} \\
\text {reten }(\mathrm{L})= & \alpha(\mathrm{L})^{+}
\end{array}
$$

Notably, on this perspective, the four classical aspectual classes are derivable as the set of logically possible cross-combinations of the four aspectual features as defined above. The corresponding properties of being initially or finally bounded, are marked by using a short binary code, with the first digit referring to the beginning, the second to the ending, and 1 and 0 indicating the presence or absence of a boundary, respectively. If we let $a$ and $o$ refer to the two boundary marking propositions inside the symbols $\alpha$ and $\omega$ we get the abstract characterizations on the very right.

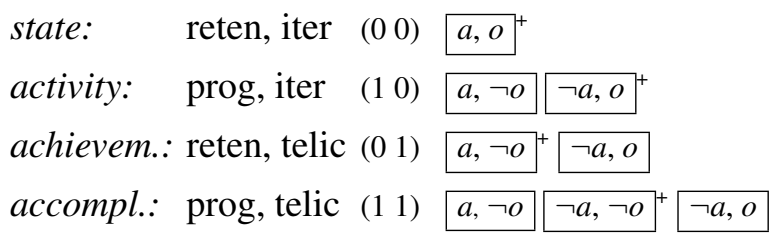

The following translations, which give formalizations within the framework of two of the initial examples, "swim" and "build a tower", may serve as a concrete illustration. ${ }^{2}$

(3) $\neg \exists \mathrm{x} \neq \emptyset(\operatorname{swim}(\mathrm{x})) \quad \exists \mathrm{x} \neq \emptyset(\operatorname{swim}(\mathrm{x}))$

(4) $\neg \exists \mathrm{x} \leq \mathrm{t}$ (build(x)) $\quad \exists \mathrm{x} \leq \mathrm{t}$ (build(x)), $\neg$ build(t) $+{ }^{+}$build(t)

\section{The Proposal}

Within the formalism introduced so far, the commonly assumed constraint on the interpretability of temporal adverbials reads as follows (where $V$ represents a verbal phrase, and $I$ an expression denoting a temporal interval):

$$
\Lambda(\mathrm{V} \text { in } \mathrm{I})=\{\Lambda(\mathrm{V}) \& \Lambda(\mathrm{I}) \text { if } \Lambda(\mathrm{V}) \text { is telic; } \emptyset \text { otherwise }\}
$$
$\Lambda(\mathrm{V}$ for $\mathrm{I})=\{\Lambda(\mathrm{V}) \& \Lambda(\mathrm{I})$ if $\Lambda(\mathrm{V})$ is iter; else $\emptyset$ otherwise $\}$.

\footnotetext{
${ }^{2}$ For the sake of abbreviation, here and in all that follows obvious entailment relations are taken into account. So, for instance, $\neg a$ is suppressed in presence of $o$ here.
} 
The aim of this section is to improve on that by giving room, within the semantic framework itself, for those kinds of adapted interpretations that actually occur, as shown by the initial examples. The approach will set the representational view (section 2) at work here, as it makes use of the possibility to introduce the concept of underspecification.

The logical heart of the proposal to be made is Supervaluation Theory, developed by van Fraassen (1969) as a formal tool for handling presupposition failure. The more general theoretical aim of van Fraassen was to account for the "third possibility" beside the classical valuations Truth and False in a way that preserves as much as possible from the classical framework. So, notably, while the supervaluationist denies the metalogical Principle of Bivalence, he still accepts the logical Law of Excluded Middle together with all other classical tautologies. This decisive difference to the several three-valued logical systems known is due to the idea of using truth-value gaps rather than a proper third value. Ordinary partial valuations are extended to supervaluations by considering the possible completions of a given model, that is the set of classical valuations such that all missing values get filled up in one way or the other.

Take metavariable $\mathrm{M}$ to stand for partial models, $\mathrm{M}^{\prime}$ to range over all possible completions of $\mathrm{M}$, and $\mathrm{M}^{*}$ to be the supermodel of $\mathrm{M}$, comprising $\mathrm{M}$ together with all its $\mathrm{M}^{\prime}$.

A supervaluation based on $\mathrm{M}$ is a function that assigns Truth with respect to $\mathrm{M}^{*}$ to a proposition $\phi$ just in case $\phi$ is classically true in all $\mathrm{M}^{\prime}$, False just in case it is false in all $\mathbf{M}^{\prime}$, and \# (gap) otherwise.

That means, if a proposition $\phi$ has received supervalue \# there are underlying, more precise models such that $\phi$ is true in some of them and false in others.

With this additional technical equipment available, let me now turn back to the problem of giving an appropriately flexible, but non-ambiguous formal characterization of the semantic contribution of temporal adverbials. The main task obviously consists in offering a proper semantics for the prepositions "in" and "for" that accords with the overall aspectual semantic formalism rather than just stipulating ungrammaticality. In order to keep the idea of a language internal criterion for the aspectual characteristics of a situation intact while allowing for the observed interpretational flexibility, those semantic characterizations should effectively constrain the basic applications of the adverbials in the way commonly assumed, but at the same time allowing and triggering compositional adaptions of sense via meaning shifts. The algorithm I am going to introduce is proceeding in three steps, illustrated here firstly by means of a telic example, and afterwards an atelic one. The general lead-in will be the idea of formally treating aspectually sensitive temporal prepositions as some kind of dynamic, structural presuppositions.

As the starting point, I assume the representations of the meanings of the two temporal prepositions "in" and "for" under (5) and (6). These formalizations take into account the known preferences with respect to the aspectual type of the situational concept to be modified by encoding the properties telic and iter according to the formal definitions given in the previous section.

$$
\text { (5) } \Lambda \text { (in) }=\neg^{+} \square \quad \text { (6) } \Lambda(\text { for })=\square \sigma^{+}
$$

In the first, preparatory step of composition, the prepositions get combined via superposition with the nominal phrase "an hour", as done below.

$$
\begin{aligned}
& \text { (7) } \Lambda(\text { for an hour })=\square^{0} 0^{+} \& \text { time(m) } \square^{+} \text {time(n), hour(m,n) } \\
& =\operatorname{time}(\mathrm{m}) \mathbf{O}^{+} \mathbf{O}, \operatorname{time}(\mathrm{n}), \operatorname{hour}(\mathrm{m}, \mathrm{n})
\end{aligned}
$$

(8) $\Lambda$ (in an hour $)=\neg 0^{+} \square^{\&}$ time(m) $\square^{+}$time(n), hour(m,n)

$$
=\neg \mathrm{O}, \text { time }(\mathrm{m}) \neg^{+} \mathrm{O}^{+} \text {time(n), hour(m,n) }
$$

Now, the adverbials are ready for modifying the situational concepts, whose abstract aspectual characterizations are given once more in (9) and (10). ${ }^{3}$ The explanation will proceed with respect to the telic example first.

$$
\text { (9) } \Lambda\left(\mathrm{V}^{\text {iter }}\right)=a, \neg o \neg \neg a, o+
$$

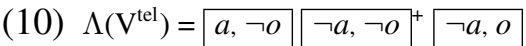

In its first, obligatory phase, the algorithm combines the representation of the temporal adverbial with the abstract characterization of the situational concept by means of superposition. If the aspectual type of the situational concept agrees with the structural condition set by the preposition, the

\footnotetext{
${ }^{3}$ Here, an activity is chosen as the atelic example, an accomplishment as the telic one. For states and achievements, though, the respective procedures would work out in exact parallel.
} 
incorporation of prepositional meaning does not have any visible effect. The semantic material just combines normally via superposition, as can be seen in (11), and the algorithm finishes here.

(11) $\Lambda\left(\mathrm{V}^{\text {tel }}\right.$ in an hour $)=$

$a, \neg o, \operatorname{time}(\mathrm{m}) \neg a, \neg O+\neg a, o, \operatorname{time}(\mathrm{n}), \operatorname{hour}(\mathrm{m}, \mathrm{n})$

However, in case of an aspectual clash between preposition and event description, combining the concepts leads to a contradiction at some predetermined position inside the complex situational type, as happens in (12). This formal accident can be traced back to the preposition and the situational type contributing different claims with respect to the truth-value of the proposition $o$, encoding the telicity feature.

(12) $\Lambda\left(\mathrm{V}^{\text {tel }} *\right.$ for an hour $)=$

$$
a, \neg o, \text { time }(\mathrm{m}) \quad \neg a, \neg O, o
$$

As these are the cases where interesting aspectual shifts turn up, the real action starts here. In its second phase, the algorithm applies a repairing mechanism by assigning a truth-value gap (\#) to the proposition that previously had received contradictory valuations. The rationale behind this may be thought of as not passing judgement in the face of equally probable but opposing evidences.

(13) $\Lambda\left(\mathrm{V}^{\text {tel \# }}\right.$ for an hour $)=$

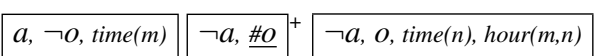

The general formal rule applied here is right the one standardly assumed for other cases of presupposition failure. That means, the meaning of a temporal preposition is thought of as some kind of dynamic, structural presupposition with respect to the development of the truth value of a certain proposition. Its semantic contribution is empty in case the verbal concept it combines with shows the right internal structure, but it causes the introduction of a truth-value gap at a particular position inside the situational string whenever its structural constraint is not satisfied. In the latter case, the result of this semantic operation is a situational concept which is underspecified with respect to the aspectual feature telic (\#telic).

As stated above, lacking a truth-value in the sense of supervaluationism, consists in the capacity in principle to make precise in more than one way. That means, for the proposition $o$ having been marked \# in a supermodel $\mathrm{M}^{*}$, there are un- derlying models in $\mathrm{M}^{\prime}$ such that $o$ is true in one of them but false in the other. This determination of previously underspecified information in all possible directions, by grounding the freshly introduced supervalue \# in the underlying classical models, is just what the algorithm is supposed to do in its third, last step. Intuitively, instead of loosing any information previously received, the ideal language user is developing different hypothetical interpretations separately. This might be taken as a strategy to save monotonicity of the interpretation process by allowing reciprocal adaptions between preposition and situation. The two obvious outputs with respect to the telic example are spelled out below.

(14) $\Lambda^{1}\left(\mathrm{~V}^{\text {tel }}\right.$ for an hour $)=$

$$
a, \neg o, \operatorname{time}(\mathrm{m}) \rightarrow \neg a, \underline{O}+\neg a, o, \operatorname{time}(\mathrm{n}), \operatorname{hour}(\mathrm{m}, \mathrm{n})
$$

(15) $\Lambda^{2}\left(\mathrm{~V}^{\text {tel }}\right.$ for an hour $)=$

$$
a, \neg o, \text { time }(\mathrm{m}) \quad \neg a, \neg O
$$

This reintroduction of truth-values after a forced gap gives rise to a specific set of new regular languages. What situational concepts do those rebuilt structures encode? In (14) the result state description appears to have spread over the entire internal part of the expression. Under this perspective the preparatory phase got abstracted away, and the event culminates immediately after start. In consequence, the adverbial now gets interpreted as indicating the duration of the result state of the situation. Obviously, this is one of the readings we had found in connection with the modification of telic situational concepts by

\begin{tabular}{|c|c|c|c|}
\hline (16) & $\begin{array}{l}\neg \exists \mathrm{x} \leq \mathrm{t}(\text { build }(\mathrm{x})) \\
\text { time }(\mathrm{m})\end{array}$ & $\begin{array}{l}\exists \mathrm{x} \leq \mathrm{t}(\text { build }(\mathrm{x})) \\
\text { build }(\mathrm{t})\end{array}$ & $\begin{array}{l}\text { build(t), } \\
\text { time }(n), \text { hour(m,n) }\end{array}$ \\
\hline
\end{tabular}
"for"-adverbials at the very beginning. Under (16) this point gets further illustrated with the help of the familiar example ("build a tower for an hour").

In contrast, the second way to make precise the aspectually underspecified concept in (13) leads up to the description under (15). Here, the preparatory phase is making up the whole inner part of the regular expression. Consequently, applying the temporal adverbial gives the description of an event that takes an hour to culminate. What we have here clearly amounts to the preparatory phase reading of the "for"-adverbial. Again, (17) offers concrete illustration.

(17)

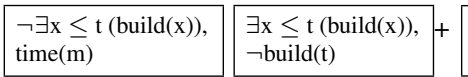
build(t), time(n), hour(m,n) 
Notice that the formal mechanism does in principal not serve to get rid of the factivity entailment for the result state here. While this seems plausible for examples like "grow old for many years", it is certainly not so for "read a book for a few minutes". I take this ambivalence as indicating that this question is not a matter of aspectual logical form, causing "hard" semantic inferences, but that it depends on background knowledge concerning default durations of certain kinds of events. With respect to the above example, this would mean that in order to eventually strip off the result state, a second, pragmatic principle would have to get applied to the intermediate, semantic output gained here.

So far so good. But what about the iterative reading we found as the third possibility when looking at this kind of example at the very beginning? To get this interpretation derived, let us take one out of the several different-sized strings encoded by the regular expression in (13), which, for instance, may look like this.

(18) $\Lambda^{\prime}\left(\mathrm{V}^{\text {tel \# }}\right.$ for an hour $)=$

\begin{tabular}{|c|c|c|c|c|}
\hline $\begin{array}{l}a, \neg o, \\
\operatorname{time}(m)\end{array}$ & $\neg a, \underline{\# O}$ & $\neg a, \underline{\# O}$ & $\neg a, \underline{\# O}$ & $\neg a, \underline{\text { \#O }}$ \\
\hline
\end{tabular}

Now, obviously, different classical valuations can be chosen for different states, leading, for instance, to the pattern under (19).

(19) $\Lambda^{3}\left(\mathrm{~V}^{\text {tel } \#}\right.$ for an hour $)=$

\begin{tabular}{|c|c|c|c|c|c|}
\hline $\begin{array}{l}\text { a, } \neg O, \\
\text { time }(m)\end{array}$ & $\neg a, \underline{o}$ & $\neg a, \underline{\neg O}$ & $\neg a, \underline{o}$ & $\neg a, \neg O$ & $\begin{array}{l}\neg a, O, \\
\text { time }(n), \operatorname{hour}(m, n)\end{array}$ \\
\hline
\end{tabular}

That means, preparatory phases freely alternate with result states, thereby forming a situational description which can only be interpreted as referring to a situation comprising several iterations of the original event. This complex situation as a whole becomes the attaching point of the temporal information carried by the adverbial. Under (20) this move is spelled out with respect to the concrete example.

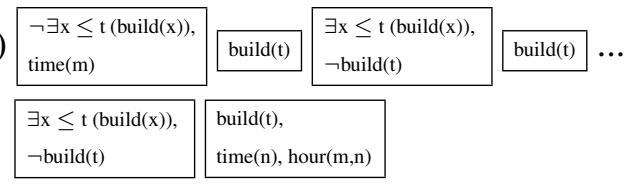

After having derived all possible interpretations of a "for"-adverbial when applied to a telic concept, the task of accounting for adapted readings of an "in"-adverbial modifying an atelic situational description is still at stake. A preparatory phase and a boundary reading is what we would wish the semantic mechanism to deliver here. The following formulas are the result of trying out the three steps of the shifting algorithm - superposition, reparation, determination - for the atelic case.

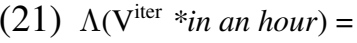

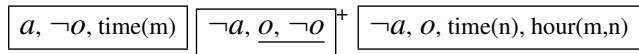

As before, in order to escape contradiction the algorithm proceeds by underspecifying the critical telicity structural information. The side effect of the truth-value gap spreading over from $o$ to $a$ in the atelic example in (22) is due to the fact of the both proposition generally being negation variants of each other here (compare (3)).

(22) $\Lambda\left(\mathrm{V}^{\text {iter \# }}\right.$ in an hour $)=$

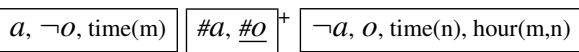

Then, fulfilling the third, determination step with respect to the above underspecified concept, leads to the following two regular languages.

(23) $\Lambda^{1}\left(\mathrm{~V}^{\text {iter }}\right.$ in an hour $)=$

$a, \neg O, \operatorname{time}(\mathrm{m}) \quad a, \neg O+\neg a, o, \operatorname{time}(\mathrm{n}), \operatorname{hour}(\mathrm{m}, \mathrm{n})$

(24) $\Lambda^{2}\left(V^{\text {iter }}\right.$ in an hour $)=$

$a, \neg o$, time $(\mathrm{m}) \quad \neg a, \underline{o}+{ }^{+} \neg a, o$, time(n), hour(m,n)

In (23) the start of the phase of activity is postponed to the very last symbol. On that basis, the temporal adverbial gets interpreted as specifying the duration of the respective pre-phase. In (24), on the other hand, the activity phase of the situation got spread over the entire real suffix of the regular expression. Therefore, the adverbial adds information concerning the temporal boundaries of the whole situation. So, both known interpretational possibilities can be accounted for by the proposed formalism. ${ }^{4}$ Again, the concrete example ("swim in an hour") may ease understanding.

\begin{tabular}{|c|c|c|}
\hline $\begin{array}{l}\neg \exists \mathrm{x} \neq \emptyset(\operatorname{swim}(\mathrm{x})), \\
\text { time }(\mathrm{m})\end{array}$ & $\neg \exists x \neq \emptyset(\operatorname{swim}(x))+$ & $\begin{array}{l}\exists x \neq \emptyset(\operatorname{swim}(x)), \\
\text { time(n), hour }(m, n)\end{array}$ \\
\hline $\begin{array}{l}\neg \exists \mathrm{x} \neq \emptyset(\operatorname{swim}(\mathrm{x})), \\
\text { time }(\mathrm{m})\end{array}$ & $\exists x \neq \emptyset(\operatorname{swim}(x))+$ & $\begin{array}{l}\exists x \neq \emptyset(\operatorname{swim}(x)), \\
\text { time(n), hour(m,n) }\end{array}$ \\
\hline
\end{tabular}

The aim of the semantic interpretation process has been achieved at this point. It was to make provision for the set of possible readings found in the cases under consideration in a fully composi-

${ }^{4}$ The formally possible third variant, parallel to the iterative interpretation gained for the telic example, is not very prominent here and usually not discussed in the literature. But there are certainly special contexts in which this description may nevertheless apply (imagine some sort of interval training session, for instance). So, there is no over-generation here. 
tional manner, i. e., only using lexical semantic entries and general rules for combining them. Further methods will have to apply in order to filter out the best actual candidate with the help of more contextual information and world knowledge.

\section{Conclusion}

The aim of this paper was to show how the meaning potential of temporal adverbials can be formally accounted for, and to give room, within the compositional semantic framework itself, for an efficient derivation and systematic explanation of the exact number and kinds of possible readings.

According to the proposal made here, aspectual shifts consist in restructuring a situational concept from inside rather than in simple mappings from one atomic event onto another. Aspectual transitions thus happen with respect to linguistic representations rather than ontological entities and are made on a finer, subatomic semantic level.

The heart of the proposal is the concept of aspectual underspecification and the idea of formally treating the meaning of temporal prepositions as some kind of dynamic, structural presuppositions. Thus, the whole process is triggered by some kind of type conflict, as traditionally assumed, but gets now controlled by the introduction and consequent filling in of a truth-value gap. Literally, the meaning of a verbal phrase including an aspectual clash between temporal adverbial modifier and situational concept to be modified is an aspectually underspecified situational concept. The possible aspectually shifted readings then appear as the different ways of specifying it.

As opposed to what is the case in traditional theories of aspect shift, those possible ways of specifying are compositionally determined, i.e. by exclusive recurrence of semantic material already present in the enriched representation, and via application of the standardly assumed rule for cases of presupposition failure. Therefore, the semantic process in question is analysed here as entirely language internal.

Another interesting feature of the proposed algorithm when compared to the traditional approach seems to be that it is not the one-sided compliance of letting the verbal phrase possibly be type-changed by the adverbial, but rather the idea of allowing reciprocal adaptations that finally leaves us with the right set of predictions. Hereby, monotonicity of the overall interpretation process is preserved.

Last but not least, the procedure of systematically introducing underspecification as some kind of claimed ignorance in the presence of conflicting evidence, and of subsequently trying out all possible specifications separately, seems to be a plausible hypothesis about the rational strategies we really may use when adapting sense.

\section{References}

de Swart, Henriette. 1998. Aspectual Shift and Coercion. Natural Language and Linguistic Theory, 16:347-385.

Fernando, Tim. 2003. Finite-state descriptions for temporal semantics. Computing Meaning, volume 3. Kluwer, Tilburg.

Fernando, Tim. 2004. A finite-state Approach to Events in Natural Language Semantics. Journal of Logic and Computation, 14(1):79-92.

Gründer, Sabine. 2008. Aspectual Shift as Supervaluation. (to appear), Available under: http : //www.asv.informatik.uni leipzig.de/opencms/opencms/asv/de/ Mitarbeiter/Sabine_Gruender.html

Moens, Marc and Steedman, Mark. 1988. Temporal Ontology and Temporal Reference. Computational Linguistics, 14(2):15-28.

Pulman, Stephen, G. 1997. Aspectual Shift as Type Coercion. Transactions of the Philological Society, 95(2):279-317.

Vendler, Zeno. 1957. Verbs and Times. Philosophical Review, 66:143-160.

van Fraassen, Bas. 1969. Presuppositions, supervaluations and free logic. in K. Lambert (ed) The Logical Way of Doing Things, 67-91. Yale University Press, New Haven. 